\section{IDDF2019-ABS-0081 PORTAL FREE CHOLESTEROL AND OXIDIZED-LDL ACCUMULATION IS ASSOCIATED WITH PLAQUE FORMATION AND INFLAMMATION IN HUMAN NAFLD}

${ }^{1}$ Cheng-Maw Ho*, ${ }^{1}$ Shu-Li Ho, ${ }^{2}{ }^{Y}$ u-Sheng Lai, ${ }^{2}$ Shao-Chun Lu, ${ }^{1}$ Hui-Ling Chen, ${ }^{2}$ PoYuan Chang, ${ }^{1}$ Rey-Heng Hu, ${ }^{1}$ Po-Huang Lee. ${ }^{1}$ National Taiwan University Hospital, Taiwan; ${ }^{2}$ National Taiwan University, Taiwan

\subsection{6/gutjnl-2019-IDDFabstracts.88}

Background Macrophage uptake of oxidized low-density lipoprotein (oxLDL) which may result in the accumulation of free cholesterol and subsequent crystal formation that can induce NLRP3 inflammasome activation. Hepatic free cholesterol content is significantly increased in human non-alcoholic steatohepatitis. We aim to investigate the roles of free cholesterol and oxLDL deposition on portal venous inflammation, atherosclerosis and fibrosis in the pathogenesis of human non-alcoholic fatty liver disease (NAFLD).

Methods Surgical specimens of NAFLD were stained with antibodies against oxLDL, $\alpha$-smooth muscle actin, lectin-like oxLDL receptor-1 (LOX-1, a scavenger receptor implicated in endothelial dysfunction and atherosclerosis), CD68, CD11b, CD31 and interleukin $1 \beta$ (IL-1 $\beta$ ). Free cholesterol was visualized by filipin stain. The display localization of oxLDL and its associated histopathological changes in serial sections were correlated with other markers.

Results Histologically, free cholesterol was co-localized with oxLDL. Their accumulation at the wall of the portal vein is associated with the narrowing of the lumen, plaque formation, endothelium deformation and periportal inflammation. Inflammation was evidenced by the co-localization of Kupffer cells $\left(\mathrm{CD}_{6} 8^{+} \mathrm{CD} 11 \mathrm{~b}^{-}\right)$and IL-1 $\beta$ and the expression of LOX-1. Notably, ruptured plaques were closely associated with portal venous inflammation. In cirrhosis, free cholesterol and oxLDL accumulation at periportal and sinusoidal areas were associated with regional stellate cell activation and chicken-wire fibrosis.

Conclusions In human NAFLD, free cholesterol and oxLDL accumulation at the portal vein was associated with plaque formation and inflammation, and inflammation was especially prominent in the ruptured plaques. Free cholesterol and oxLDL accumulation in the cirrhotic parenchyma was associated with chicken-wire fibrosis, probably through stellate cell activation. These findings provide not only pathogenic corroboration of atherosclerosis in liver but a direct link between NAFLD and coronary artery disease.

\section{IDDF2019-ABS-0102 COMPARISON OF MURINE STEATOHEPATITIS MODELS IDENTIFIES A DIETARY INTERVENTION WITH ROBUST FIBROSIS, DUCTULAR REACTION AND RAPID PROGRESSION TO CIRRHOSIS, CANCER}

${ }^{1}$ Guangyan $\mathrm{Wei}^{*}$, ${ }^{2}$ Ping An, ${ }^{2}$ Kahini Vaid, ${ }^{2}$ Imad Nasser, ${ }^{2}$ Pinzhu Huang, ${ }^{2}$ Shuangshuang Zhao, ${ }^{2}$ Detlef Schuppan, ${ }^{2}$ Yury Popov. ${ }^{1}$ The First Affiliated Hospital of Sun Yat-sen University, China; ${ }^{2}$ Beth Israel Deaconess Medical Center, Harvard Medical School, USA

\subsection{6/gutjnl-2019-IDDFabstracts.89}

Background Progressive fibrosis, functional liver failure and liver cancer are the central liver-related outcomes of nonalcoholic steatohepatitis (NASH). However, these pathologies are notoriously difficult to achieve in mouse models. We, therefore, performed a direct, quantitative comparison of hepatic fibrosis progression in well-defined methionine- and cholinedeficient (MCD) and choline-deficient, amino-acid defined (CDAA) diets with various fat content $(10-60 \%$ by calories) in $\mathrm{C} 57 \mathrm{Bl} / 6 \mathrm{~J}$ and $\mathrm{BALB} / \mathrm{c}$ mice.

Methods MCD or CDAA diets were formulated with a fat content ranging from 10 to $60 \%$ by calories, and fed C57Bl6 and $\mathrm{BALB} / \mathrm{c}$ mice for up to 24 weeks. Hepatic fibrosis progression was assessed using standardized histological and biochemical methods.

Results MCD feeding resulted in moderate fibrosis with an up to 2-fold increase in total hepatic collagen content as determined via hydroxyproline (HYP) at week 8 and progressive weight loss, irrespective of dietary fat. In contrast, CDAA-fed mice did not lose weight and developed fibrosis starting from week 4. Dietary fat intake dose-dependently increased the ductular reaction and fibrosis in $\mathrm{C} 57 \mathrm{Bl} / 6 \mathrm{~J}$ mice on the CDAA diet. Longitudinal analysis of CDAA with $60 \%$ fat (HF-CDAA) feeding revealed pronounced ductular reaction and perisinusoidal bridging fibrosis $(\mathrm{C} 57 \mathrm{Bl} / 6 \mathrm{~J}>\mathrm{BALB} / \mathrm{c})$, with a 7-fold HYP increase at week 12, that showed limited spontaneous reversibility. At 24 weeks, HF-CDAA mice developed signs of cirrhosis with severe pan-lobular 'chicken wire' fibrosis, 10-fold HYP increases, regenerative nodules, portal hypertension and elevated serum bilirubin and ammonia levels; $80 \%$ of mice $(8 /$ 10) developed multiple glypican-3- and/or glutamine synthetase-positive hepatocellular carcinomas (HCC).

Conclusions In conclusion, the 60\% HF-CDAA feeding in $\mathrm{C} 57 \mathrm{Bl} / 6 \mathrm{~J}$ mice was identified as an optimal model of steatohepatitis with robust fibrosis and ductular proliferations that progress to cirrhosis and HCC within 24 weeks. This robust model will aid the testing of interventions and drugs for severe NASH.

\section{IDDF2019-ABS-0117 STUDY ON VALIDITY OF BIOMARKERS DKK1 AND HBX-LINE1 IN DIAGNOSIS AND POSTTREATMENT MONITORING OF HEPATOCELLULAR CARCINOMA}

${ }^{1}$ Hieu Le Trung*, ${ }^{2}$ Hai Le Trung. ${ }^{1} 108$ Military Central Hospital, Vietnam; ${ }^{2}$ Hanoi College of Medicine and Pharmacy, Vietnam

\subsection{6/gutjnl-2019-IDDFabstracts.90}

Background Evaluate the validity of DKK1 and HBx-LINE1 in diagnosis and post-treatment of $\mathrm{HCC} / \mathrm{HBV}$ and analyse relationship with clinical and paraclinical some characteristics.

Methods Study on 114 HCC patients at Central Military Hospital 108, Military Hospital 103 and Military Hospital 175 (1/2016-3/2018) with DKK1 and HBx-LINE1, re-examination after surgery.

Results With DKK1 $\geq 2,15 \mathrm{ng} / \mathrm{mL}$, the positive rates of serum protein DKK1 were significantly increased when compared with those of AFP $(97.37 \%$ so voi $62.92 \%)$. The mean of serum protein DKK1 of HCC was significantly higher than it in liver cirrhosis patients with $\mathrm{p}<0.05$. The combination between AFP and DKK1 expression will improve positive rates and help more diagnosis in $12.3 \%$ of HCC cases. Logistic regression analysis showed the risk of HCC will increase about 18.5 times when DKK1 $\geq 2,15 \mathrm{ng} / \mathrm{mL}$. 